\title{
Effect of Surface Defects and Internal Pressure on Fatigue Life of Coiled Tubing
}

\author{
Bing Liu \\ College of Mechanical and Electronic \\ Engineering, \\ Shandong University of Science and \\ Technology \\ Qingdao, China \\ liubing5195@163.com \\ Baokun Han \\ College of Mechanical and Electronic \\ Engineering, \\ Shandong University of Science and \\ Technology \\ Qingdao, China \\ Bk_han@163.com
}

\author{
Tao Li \\ College of Mechanical and Electronic \\ Engineering, \\ Shandong University of Science and \\ Technology \\ Qingdao, China \\ 1343471869@qq.com \\ Huitao Zhuang \\ Suzhou Douson Drilling\&Production \\ Equipment Co.,Ltd \\ Suzhou, China \\ Zhuang_Huitao@douson.cn
}

\author{
Liping Xu \\ College of Mechanical and Electronic \\ Engineering, \\ Shandong University of Science and \\ Technology \\ Qingdao, China \\ 499328331@qq.com \\ Shulin $\mathrm{Li}$ \\ Suzhou Douson Drilling \&Production \\ Equipment Co.,Ltd \\ Suzhou, China \\ Li_Shulin@douson.cn
}

\begin{abstract}
In the Complex service environment, the surface of coiled tubing has different degrees of defects, which can easily lead to the stress concentration on the surface of coiled tubing and cause premature fatigue failure of the tubing. In order to estimate the fatigue life of coiled tubing more accurately, and ensure the stability and safety of coiled tubing, it is necessary to consider the influence of surface defects on the fatigue life of coiled tubing. The fatigue test of QT800 coiled tubing is carried out under the condition of $0-7000 \mathrm{psi}$ internal pressure. The research shows that: (1) With the increase of internal pressure, the fatigue life of coiled tubing decreases significantly. When the internal pressure reaches $4000 p s i$, the fatigue life of coiled tubing is reduced by about $80 \%$.(2) The influence degree from large to small of surface defect on coiled tubing fatigue life is: circumferential defect, square pit defect and axial defect.(3) The fatigue life prediction model of coiled tubing with surface defects is obtained by local strain method, and the average error of theoretical value and experimental value is $8.85 \%$, which can meet the requirements of engineering precision.
\end{abstract}

Keywords-coiled tubing, surface defects, internal pressure, fatigue life, local strain method

\section{INTRODUCTION}

Compared with conventional thread connecting pipe, coiled tubing has the advantages of quick operation, high efficiency, safety and reliability [1]. It has been widely used in oil and gas field drilling, well completion, oil recovery and other operations [2-3]. During the operation of coiled tubing, the stress state of the coiled tubing is complex. It not only suffers from many plastic bending deformations, but also is subjected to internal pressure, so the fatigue life of the coiled tubing is greatly limited [4-5]. At the same time, the service environment of coiled tubing is rugged, and it is easy to cause the volumetric defects such as depression, incision and so on, which cause mechanical damage or corrosion on the coiled tubing surface. The stress concentration in the defect zone will aggravate the crack propagation and lead to premature fatigue failure of coiled tubing [6-7].
The ultra-low cycle fatigue behavior of coiled tubing makes it very important to study its fatigue prediction. A large number of coiled tubing fatigue life prediction models have been developed all over the world. The United States Tipton[8-10], according to a large number of experimental results for the study of multiaxial low cycle fatigue failure theory, based on the fatigue damage accumulation theory of linear Miner established a life prediction model, found that the coiled tubing internal pressure under the condition of small prediction results are satisfactory. The stress analysis and strength check of the pre bending coiled tubing were carried out by Professor Li Zifeng [11] of Yanshan University. The stress cycle characteristics of the pre bending coiled tubing were analyzed in the field operation. On the basis of the fatigue test data under symmetrical cyclic and pulsating cycles, a mathematical model for predicting the fatigue life of pre bending coiled tubing under arbitrary cyclic conditions is established by using the mathematical method of fitting and interpolation. Wang Youqiang [12] of Qingdao Institute of architectural engineering and Zhang Siwei of China University of Petroleum converted the low cycle fatigue strain life relationship of coiled tubing into the stress life relationship, and obtained 1.5 empirical life formula. The probability distribution model of fatigue life of coiled tubing is determined by using fuzzy Bayes theory, and the prediction model of fatigue life and the main factors affecting the fatigue life are obtained. In this study, coiled tubing with surface defects was studied, and the fatigue life model of coiled tubing was established to provide guidance for field construction of coiled tubing.

\section{Fatigue Life Test of CoIled Tubing}

Coiled tubing fatigue life test is divided into 2 sets of experiments, which are divided into no surface defects and fatigue tests with surface defects. The fatigue life test of coiled tubing with no surface defect is divided into no internal pressure and internal pressure, the pressure grade is 0-7000psi, and 3 specimens of each pressure grade are tested. The fatigue life test of coiled tubing with surface defects is divided into three kinds, including axial defect, 
circumferential defect and square pit defect. The pressure level is $0-7000$ psi.

The tube specimen selected for coiled tubing fatigue life test is taken from finished product QT800 coiled tubing (2.375" diameter and 0.156" wall thickness), the length of the fatigue specimen is $60 "$ ". The performance of the material is shown in Table 1 [13].

Tab 1 Parameters of coiled tubing test parts

\begin{tabular}{cccc}
\hline Specimen type & $\begin{array}{c}\text { Young's modulus } \\
\text { E/Gpa }\end{array}$ & $\begin{array}{c}\text { Poisson ratio } \\
/ \mu\end{array}$ & $\begin{array}{c}\text { Yield strength } \\
\sigma_{s} / \mathrm{Mpa}\end{array}$ \\
\hline QT-800 & 210 & 0.3 & 552 \\
\hline
\end{tabular}

In order to simulate the defects on the surface of coiled tubing during actual service, artificial defects were manufactured on the surface of coiled tubing, as shown in Figure 1.

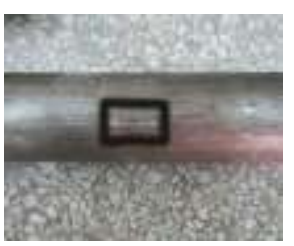

(a) Axial defect

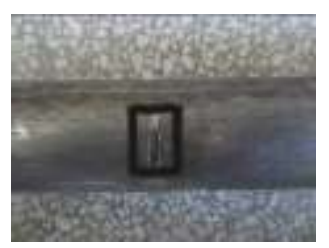

(b) Circumferential defect

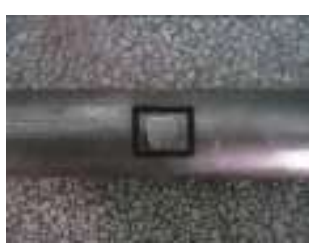

(c) Square pit defect
Fig.1. Artificial defect damage of coiled tubing test specimen

\section{A. The Effect of Internal Pressure on Fatigue Life}

The pressure test of coiled tubing without surface defect is carried out, and the low cycle fatigue life of coiled tubing under different internal pressure conditions is explored, as shown in Figure 2. From Fig.2, we can see that with the increase of coiled tubing internal pressure, the low cycle fatigue life of coiled tubing decreases. When the internal pressure of the coiled tubing is increased to 2000psi, the fatigue life of the coiled tubing decreases about $40 \%$ compared to the non internal pressure. With the internal pressure increasing to high pressure of $4000 \mathrm{psi}$, the fatigue life is only $20 \%$ of the non internal pressure condition, and the internal pressure seriously affects the service life of the coiled tubing. When the internal pressure is greater than 4000 psi, the decreasing trend of the fatigue life of coiled tubing tends to be slow, and the life remaining of the coiled tubing is little, about $10 \%$ of the fatigue life without internal pressure.

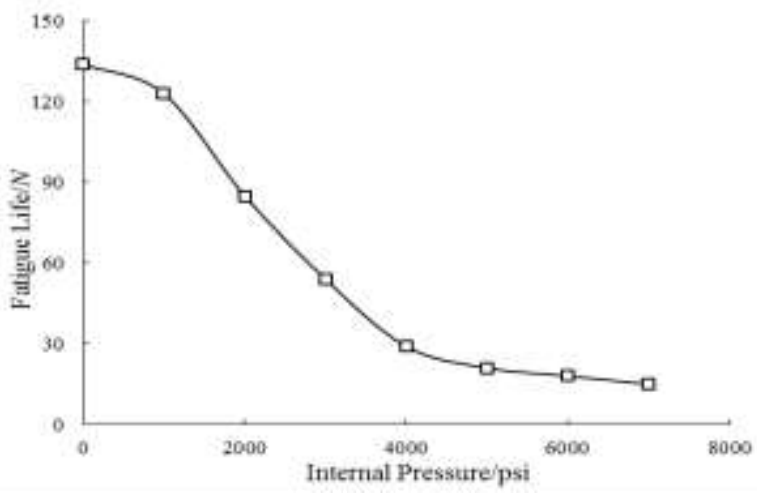

Fig.2. Effect of internal pressure on fatigue life of coiled tubing

\section{B. The Effect of Surface Defects on Fatigue Life}

When the defect length $x=0.125$ "and the defect width $w=0.125 "$, the fatigue life of the coiled tubing with different depth of the defects is explored in different internal pressure, and the effect of the surface defect depth on the low cycle fatigue life of the coiled tubing is obtained, such as Figure 3. From Figure 3, it is found that when the depth of the surface defect reaches about $40 \%$ of the coiled tubing wall thickness, the fatigue life of the coiled tubing at low pressure decreases by about $70 \%$ compared to the non surface defect. Surface defects seriously affect the service life of coiled tubing, so we should try to avoid further enlargement of the surface defects of coiled tubing. In the high pressure area, the effect of surface defects on the fatigue life of coiled tubing is less. It is found that the main cause of this phenomenon and the formation of the crack are related to the expansion. In the case of no surface defects, the surface crack of the coiled tubing is relatively long under low pressure, and the crack formation period takes a large proportion in the whole fatigue life, so the effect of surface defects is obvious. Under high pressure, the formation cycle of the coiled tubing surface crack is short, and the effect of surface defects on the fatigue life of coiled tubing is relatively small [8]. Therefore, the effect of surface defects on the fatigue life of coiled tubing under high pressure is smaller than that of low pressure. If the effect of corrosion and acidification is taken into account, the fatigue life of the coiled tubing will be reduced, which leads to the unavailability of the internal pressure of the coiled tubing to the high pressure area.

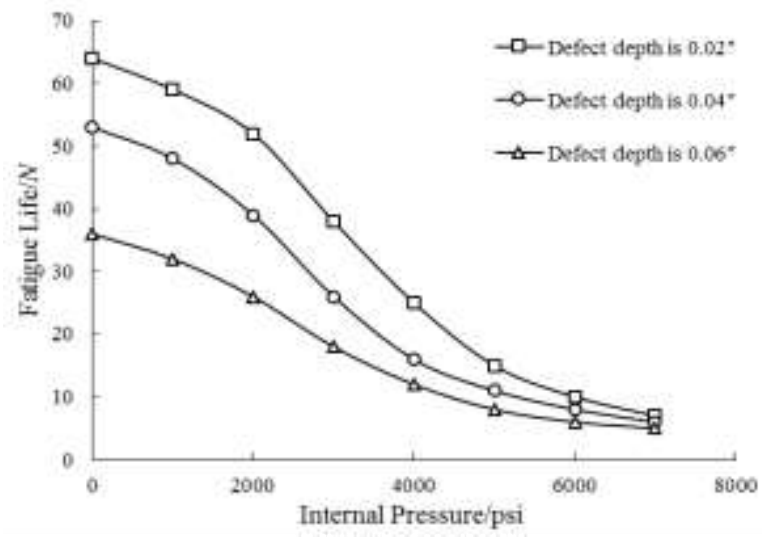

Fig.3. Effect of defect depth on fatigue life of coiled tubing

When the defect length is $x=0.125$ "and the defect depth is $d=0.02$ ", the influence of the width of the surface defect on the low cycle fatigue life of coiled tubing is analyzed, as shown in Figure 4. In the low pressure area, when the width of the surface defect reaches $15 \%$ of the diameter of coiled tubing, the fatigue life decreases to about $50 \%$ of the previous experiment. With the increase of the width of the surface defect, the fatigue life of the coiled tubing decreases as well. When the width of the surface defect reaches about $30 \%$, the fatigue life is only about $25 \%$ of the initial fatigue life. Therefore, when the width of the coiled tubing surface defect reaches the $15 \%-30 \%$ of the pipe diameter, it is necessary to pay strict attention to the use of the coiled tubing, or to cut off the coiled tubing body, so as to reduce the impact on the overall life of the coiled tubing. 


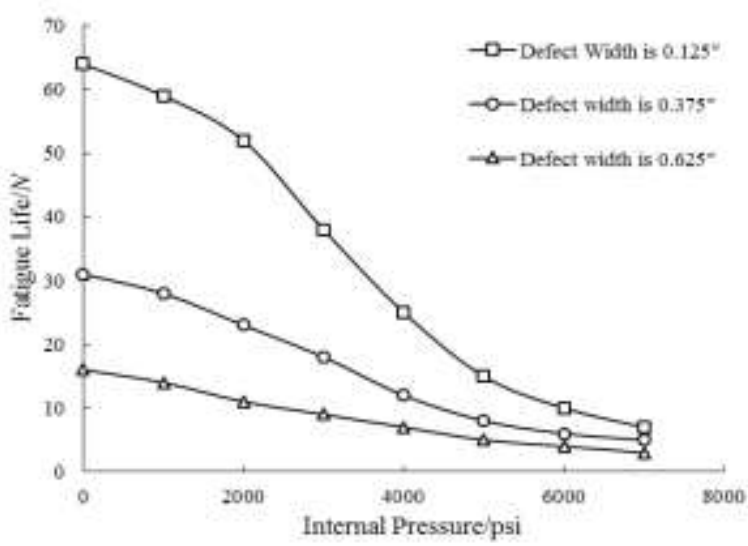

Fig.4. Effect of defect width on fatigue life of coiled tubing

When the defect depth is $d=0.02$ "and the defect width is $w=0.125 "$, the influence of the length of the surface defect on the life of coiled tubing is analyzed, as shown in Figure 5. With the increase of the length of the defect, the service life of the coiled tubing is slightly increased in the low pressure area, because with the increase of the surface defect length, the strain concentration factor of the coiled tubing becomes smaller, so the fatigue life of coiled tubing will be increased slightly.

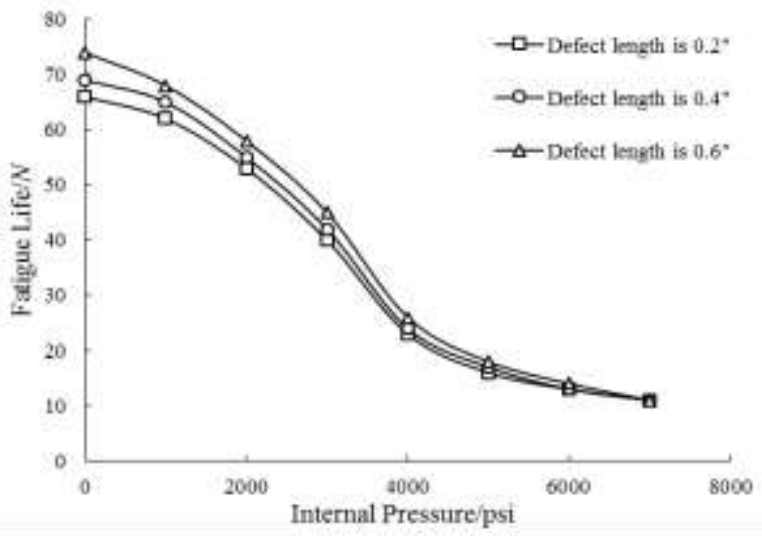

Fig.5. Effect of defect length on fatigue life of coiled tubing

Through experimental comparison, it is found that the fatigue life of coiled tubing decreases with the increase of internal pressure. When the internal pressure reaches 4000psi, the fatigue life of the specimen without internal pressure coiled tubing is reduced by about $80 \%$. By comparing the fatigue life results of the coiled tubing and surface defect damage specimens with no surface defects in low pressure area, it is found that the surface defects have a great influence on the fatigue life of the coiled tubing. The influence degree from large to small of surface defect on coiled tubing fatigue life is: circumferential defect, square pit defect and axial defect.

\section{FATIGUE LiFE PREDICTION OF COILED TUBING WITH SURFACE DEFECTS}

In engineering application, the bending radius of coiled tubing is much smaller than that of permissible bending radius, and the stress concentration caused by the defect causes the coiled tubing into elastoplastic state, and the relationship between stress and strain is nonlinear, which leads to the plastic strain becoming the main factor affecting the fatigue life of the coiled tubing. In fact, the maximum local strain is determined for the coiled tubing fatigue life. Therefore, the local strain method is applied to predict the fatigue life [14].

When the coiled tubing has cyclic bending under constant bending radius and internal pressure, its stress concentration factor $\left(K_{\sigma}\right)$ is:

$$
K_{\sigma}=\frac{\varepsilon_{e}}{\varepsilon_{x}}
$$

In the type: $\varepsilon_{\mathrm{e}}$ stands for the equivalent bending strain of coiled tubing under non internal pressure condition; $\varepsilon_{\mathrm{x}}$ stands for the actual bending strain of coiled tubing under internal pressure condition.

The fatigue life of coiled tubing is affected by the axial mean bending strain amplitude caused by bending and straightening, and the effect of the mean circumferential stress caused by internal pressure. The local strain method proposed in this paper is based on the fatigue life data of the coiled tubing based on the extremely low internal pressure. Therefore, it can be assumed that the average bending strain amplitude is the only factor affecting the fatigue life of the coiled tubing. The stress concentration factor $\left(K_{\sigma}\right)$ is expressed by the ratio of circumferential stress to yield strength $\left(\sigma_{\mathrm{h}} / \sigma_{\mathrm{s}}\right)$. As shown in Figure 6.

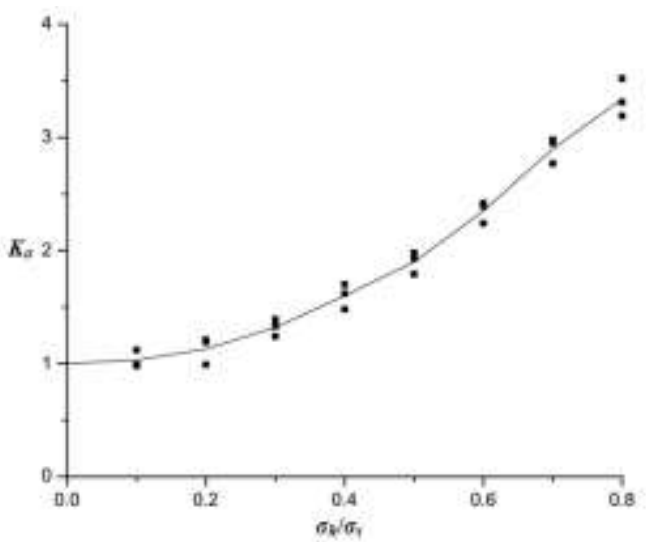

Fig.6. Fitting diagram of pressure concentration factor $\left(K_{\sigma}\right)$

The geometric relationship between the stress concentration factor $\left(K_{\sigma}\right)$ and the ratio of the circumferential stress to the yield strength $\left(\sigma_{\mathrm{h}} / \sigma_{\mathrm{s}}\right)$ is obtained by fitting with the data processing software Origin.

$$
K_{\sigma}=1+a_{1} \cdot\left(\frac{\sigma_{h}}{\sigma_{s}}\right)^{b_{1}}
$$

In the type: $a_{1}, b_{1}$ is the empirical constant of pressure concentration factor of coiled tubing. $a_{1}=3.9616, b_{1}=2.0908$.

The strain concentration factor $\left(K_{\varepsilon}\right)$ is defined as the ratio of effective strain range at the root of the defect, which is related to the defect life and the equivalent strain range:

$$
K_{\varepsilon}=\frac{\varepsilon_{d}}{\varepsilon_{e}}
$$

In the type: $\varepsilon_{\mathrm{d}}$ stands for the equivalent bending strain of coiled tubing with surface defects under internal pressure condition; $\varepsilon_{\mathrm{e}}$ stands for the equivalent bending strain of coiled tubing under non internal pressure condition. 
For the calculation of low cycle fatigue life of coiled tubing with surface defects, it is necessary to consider the influence of the shape and size of surface defects. Therefore, the surface defect severity parameter $Q$ is introduced to express the effect of surface defects on the fatigue life of coiled tubing [15]:

$$
Q=\left[\frac{d}{s} \cdot \frac{w}{x} \cdot \sqrt{\frac{A_{p}}{A_{c}}}\right]^{\frac{1}{3}}
$$

In the type: $d$ is the depth of surface defects of coiled tubing; $s$ is the wall thickness of coiled tubing; $w$ is the width of surface defects of coiled tubing; $\mathrm{x}$ is the length of surface defects of coiled tubing; $A_{\mathrm{p}}$ is the projection area of coiled tubing surface defects to cross section.; $A_{\mathrm{c}}$ is the bearing section area of the coiled tubing at the surface defect.

Through the experimental data of coiled tubing low cycle fatigue, we can get the relationship between the defect severity parameter $Q$ and the strain concentration factor $\left(K_{\varepsilon}\right)$, as shown in Figure 7.

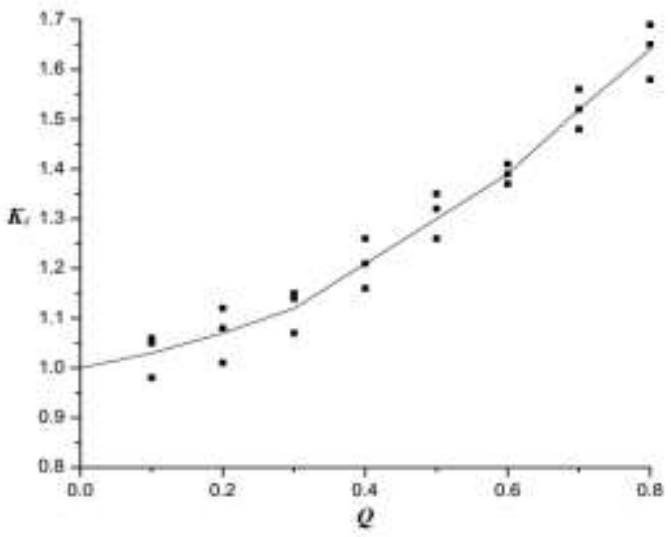

Fig.7. Fitting diagram of strain concentration factor $\left(K_{\varepsilon}\right)$

With the help of data processing software Origin, the geometric relationship between strain concentration factor and defect severity parameter $Q$ is obtained:

$$
K_{\varepsilon}=1+a_{2} \cdot Q^{b_{2}}
$$

In the type: $a_{2}, b_{2}$ is the empirical constant of strain concentration factor of coiled tubing. $a_{2}=0.96, b_{2}=1.642$.

Fatigue life prediction of QT800 coiled tubing with surface defects can be obtained through Manson-Coffin formula [16] and formula (1), (3):

$$
\varepsilon_{d}=K_{\sigma} \cdot K_{\varepsilon} \cdot \varepsilon_{x}=c \cdot N^{b}
$$

In the type: $c, b$ is the material parameters of coiled tubing with surface defects $c=0.1052, b=-0.3348$.

\section{ERROR ANALYSIS OF PREDICTION MODEL}

The prediction model is used to calculate the coiled tubing with the defect length $x=0.125 "$, the defect width $w=0.125^{\prime \prime}$ and the defect depth $d=0.02 "$. At the same time, compared with the low cycle fatigue test, the contrast curve is shown in Figure 8, and the contrast results are shown in Table 2.

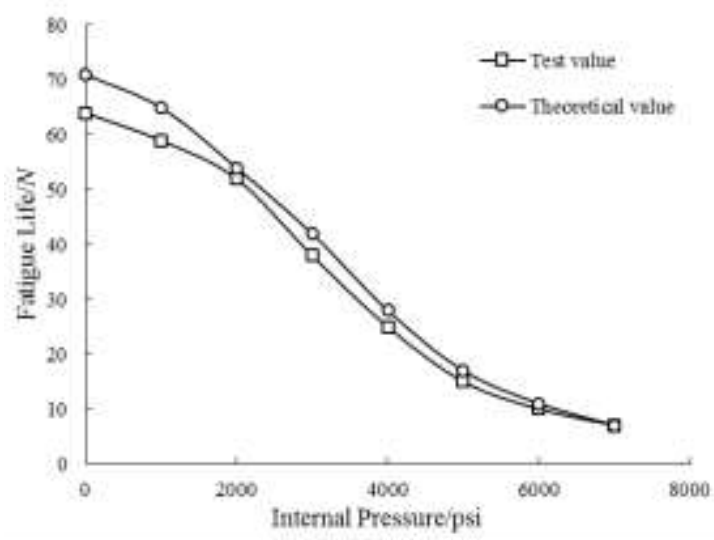

Fig.8. Curve diagram of fatigue life theoretical and test value of coiled tubing

Tab.2 Comparison of fatigue life prediction and test results of coiled tubing

\begin{tabular}{cccc}
\hline Internal pressure & \multicolumn{2}{c}{ Fatigue life } & Relative error \\
\cline { 2 - 3 }$/$ psi & Theoretical value & Test value & $1 \%$ \\
\hline 0 & 71 & 64 & 10.94 \\
1000 & 65 & 59 & 10.17 \\
2000 & 54 & 52 & 3.85 \\
3000 & 42 & 38 & 10.53 \\
4000 & 28 & 25 & 12.00 \\
5000 & 17 & 15 & 13.33 \\
6000 & 11 & 10 & 10.00 \\
7000 & 7 & 7 & 0.00 \\
\hline
\end{tabular}

The calculation results show that under given conditions, the fatigue life of the coiled tubing test and the theoretical fatigue life is basically consistent, the maximum error is $13.33 \%$, the average error is $8.85 \%$, and it is within the range of engineering precision.

\section{CONCLUSION}

(1) The experimental results show that the fatigue life of the coiled tubing is seriously affected by the internal pressure when it ranges from 1000 to 4000psi. When the internal pressure reaches $4000 \mathrm{psi}$, the fatigue life of the coiled tubing is only $20 \%$ of the non internal pressure condition.

(2) It is found that when the depth of the surface reaches about $40 \%$ of the wall thickness of the coiled tubing, the fatigue life is reduced by about $70 \%$ compared with that without the surface defect under low internal pressure. When the width of the surface defect reaches $30 \%$ of the diameter of the coiled tubing, the fatigue life is only about $25 \%$ of that without defects. The increase of surface defect length makes the strain concentration factor of coiled tubing smaller, and it will increase the low cycle fatigue life of coiled tubing. The influence degree from large to small of surface defect on coiled tubing fatigue life is: circumferential defect, square pit defect and axial defect.

(3) Using the established life prediction model to calculate the coiled tubing with defect length $x=0.125 "$, defect width $w=0.125$ " and defect depth $d=0.02 "$, the fatigue life of coiled tubing test and theoretical fatigue life is basically consistent, the maximum error is $13.33 \%$, the average error is $8.85 \%$, and it can meet the requirements of engineering precision. 


\section{ACKNOWLEDGMENT}

his study was financially supported by the Postdoctoral Research Funding Project of Jiangsu Province(1601053C); China national coal association science and technology research guidance project(MTKJ2016-279); and Shandong Province key research and development projects (2017GGX40120).

\section{REFERENCES}

[1] He Huiqun, Wang Jinhong, Chang Min, et al. The distribution and growth of Global coild tubing device [J]. Petroleum machinery, 2011, 39 (4): 77-79.

[2] Hu Yueting. Horizontal section length optimization method [J]. Acta petrolei Sinica, 2000, 21 (4): 80-86.

[3] Wang Haitao, Li Xiangfang. The coiled tubing technique in underground work in the application and thinking of $[\mathrm{J}]$. Oil drilling technology, 2008, 30 (6): 120-124.

[4] Perry K F. Microhole Coiled Tubing Drilling: A Low Cost Reservoir Access Technology [J]. Journal of Energy Resources Technology, 2007, 131(1):849-860.

[5] Yan Xiangzhen, Deng Weidong, Gao Jinwei, et al. In casing drilling and fatigue reliability of casing string mechanical properties $[\mathrm{J}]$. Acta petrolei Sinica, 2009, 30 (5): 769-773.
[6] Padron T, Luft B H, Kee E, et al. Fatigue Life of Coiled Tubing With External Mechanical Damage[C]// 2007.

[7] Adrichem W P V. Coiled Tubing Failure Statistics Used to Develop Tubing Performance Indicators [J]. Spe Drilling \& Completion, 1999, 17(3):173-182.

[8] Tipton S M, Neuharth L G, Sorem J R. Influence of Prior Cycling on Fatigue Damage Caused By in Coiled Tubing[J]. SPE, 2006, 100199.

[9] Tipton S M, Brown P A. Monitoring coiled tubing fatigue life[C]//International Conference on Coiled Tubing Technology, Houston, Texas, USA. 1994: 29-31.

[10] Tipton S M, Nelson D V. Advances in multiaxial fatigue life prediction for components with stress concentrations [J]. International Journal of Fatigue, 1997, 19(6): 503-516.

[11] Li Zifeng, Li Xuejiao, Wang Peng. Prebening coiled tubing and fatigue life Prediction [J]. Acta Petrolei Sinica, 2012, 33 (4): 706-710.

[12] Wang Youqiang, Zhang Siwei. Research progress of coiled tubing reliability [J]. Petroleum machinery, 1998 (6): 50-53.

[13] Zhao Zhangming. Coiled tubing engineering technical manual [M]. Beijing: Petroleum Industry Press, 2011:117.

[14] Zhao Shaobian, Wang Zhongbao, fatigue design, [M]. Machinery Industry Press, 1992.

[15] Christian A, Tipton S M. Statistical Analysis of Coiled Tubing Fatigue Data[C]//SPE/ICoTA Coiled Tubing \& Well Intervention Conference and Exhibition. Society of Petroleum Engineers, 2009.

[16] Chen Daiheng. Fatigue failure and material strength prediction: the concept and application of linear notch mechanics [M]. Tsinghua University press, 2014, 135. 